\title{
Red de Centros de Documentación y Bibliotecas de Mujeres: cooperación entre las bibliotecas feministas españolas
}

\author{
Por Ana M. Muñoz-Muñoz y Montse Argente-Jiménez
}

\begin{abstract}
Resumen: La Red de Centros de Documentación y Bibliotecas de Mujeres, creada formalmente en 1995, vincula y coordina a 41 unidades de información del estado español pertenecientes a diversas entidades, y cuyos fondos documentales están especializados en estudios de género, feminismos y mujeres en general. Su finalidad es potenciar el apoyo profesional entre su personal, la cooperación bibliotecaria, la solución a problemas comunes y la elaboración de herramientas de trabajo que faciliten la labor de los centros. Se presentan sus orígenes, su evolución y desarrollo, su estructura y funcionamiento, además de un análisis DAFO que muestra la situación externa de la Red y las características internas de la misma.

Palabras clave: Bibliotecas de mujeres, Centros de documentación de mujeres, Bibliotecas especializadas, Redes de cooperación, Documentación feminista, Análisis DAFO, Redes de bibliotecas, Servicios cooperativos.
\end{abstract}

Title: Women's Documentation Centres and Libraries Network: cooperation between Spanish feminist libraries

Abstract: The Women's Documentation Centres and Libraries Network, formally established in 1995, links and coordinates 41 Spanish information units which are associated to various entities and are characterised by collections specializing in gender studies, feminism and women in general. Its purpose is to strengthen professional support for staff, library cooperation, solutions to common problems and the development of tools to facilitate the work of these centres. We present its origins, plus a SWOT analysis that shows the external situation of the network and its internal features.

Keywords: Women's libraries, Women's documentation centers, Special libraries, Cooperation networks, Documentation on Gender studies, SWOT analysis, Libraries' network, Cooperative services.

Muñoz-Muñoz, Ana M.; Argente-Jiménez, Montse. "Red de Centros de Documentación y Bibliotecas de Mujeres: cooperación entre las bibliotecas feministas españolas". El profesional de la información, 2010, septiembre-octubre, v. 19, n. 5, pp. 504-509.

DOI: $10.3145 /$ epi.2010.sep.09

\section{Introducción}

LOS ORÍGENES DE LAS BIBLIOTECAS, archivos y centros de documentación e información sobre mujeres en Europa discurren en paralelo a la historia de los movimientos sufragistas y de sensibilización con los derechos de las mujeres de finales del siglo XIX.

Estos colectivos, conscientes de que los historiadores -en masculino- pasarían por alto las acti- vidades femeninas, se conciencian de la necesidad de preservar en un espacio propio la documentación y archivos generados por ellas mismas. Con el fin de conservar estos documentos e informaciones que servirían como fuente para trazar la historia del movimiento de mujeres se crean, coincidiendo ya con la primera ola del feminismo, diversos precedentes de las actuales unidades de información sobre mujeres que todavía hoy siguen en funcionamiento: The Women's Library fundada en Londres en 1926, la Bibliothèque Marguerite Durand -París, 1931- y el International Information Centre for the Women's Movement, conocido actualmente como Aletta, Institute for Women's History creado en Amsterdam en 1935 (Anitua-Vallés et al., 2007). Unos años antes, en 1909, ligada a uno de los proyectos del feminismo social que promovía la educación de las mujeres y su incorporación al 
mercado laboral nacía en Barcelona la Biblioteca Francesca Bonnemaison, convirtiéndose así en la primera biblioteca de España y Europa dirigida a las mujeres.

http://www.londonmet.ac.uk/thewo menslibrary/

http://www.paris.fr/portail/pratiquel Portal.lut?page_id=7937\&docu ment_type_id=4\&document_id= $30863 \&$ portlet_id $=18275 \&$ multilev eldocument_sheet_id $=6916$

http://www.iiav.nl/

http://w3.bcn.es/V51/Home/V51Ho meLinkPl/0,3989,526640750,00. html

Por lo que respecta a las bibliotecas de mujeres de segunda generación, no se empiezan a crear en Europa hasta las décadas de 196070. En cuanto al Estado español, su aparición tiene lugar a finales de los años 70, al amparo de la nueva situación política y fruto del interés de asociaciones e instituciones, como receptoras de una demanda social preexistente, que iniciarán la formación de los primeros fondos documentales sobre mujeres en organizaciones como: los colectivos y asociaciones del movimiento feminista, los centros de investigación universitaria y, ya entrada la década de 1980, los organismos gubernamentales promotores de políticas públicas de igualdad entre mujeres y hombres.

\section{Red de Centros de Documentación y Bibliotecas de Mujeres}

Entre los centros de documentación y bibliotecas de mujeres españoles no hubo ningún tipo de vínculo ni coordinación hasta 1993 , en que la documentalista y activista feminista Maite Albiz, en el marco de las Jornadas Feministas Estatales Juntas y a por Todas, celebradas en Madrid, organiza un taller sobre cómo gestionar fondos documentales sobre mujeres. Allí coinciden algunas de las profesionales que trabajaban en estos centros y una de las conclusiones a que llegaron fue la necesidad de poner en común experiencias y problemas similares para establecer una colaboración duradera y estable. Al año siguiente, el Centro de Documentación y Biblioteca de Mujeres del Instituto de Promoción y Estudios Sociales (IPES), de Pamplona, decide organizar el I Encuentro de Centros de Documentación y Bibliotecas de la Mujer (Argente-Jiménez, 2008) para continuar los tímidos contactos iniciados en Madrid, conocer el estado de la cuestión y establecer relaciones con otros profesionales de España cuyos objetivos e inquietudes fuesen similares. A la convocatoria asistieron únicamente ocho centros de diferentes características y estatus, pero se constató que a pesar de contener importantes recursos documentales de calidad, eran entidades poco conocidas en su ámbito, incluso entre los profesionales usuarios de esta información. Resultó evidente que había que unir esfuerzos para promocionar la función de estas unidades de información sobre mujeres y el encuentro de Pamplona finalizó con el compromiso, por parte de la $\mathrm{Bi}$ blioteca de Mujeres (hoy absorbida por el Instituto de la Mujer del Ministerio de Igualdad), de organizar un segundo encuentro en Madrid en 1995.

\section{"Hasta 1993 no hubo ningún tipo de vínculo ni coordinación entre los centros de documentación y bibliotecas de mujeres españoles"}

En éste, que ya contó con la participación de catorce centros, (entre ellos los pertenecientes a los principales organismos de igualdad de España: Instituto de la Mujer, Instituto Andaluz de la Mujer, Insti- tut Català de les Dones y Emakunde) se toman dos decisiones básicas para el futuro de estas bibliotecas y centros de documentación:

- elaboración de un registro o directorio de unidades de información especializadas en el tema que permita visibilizarlas (De-Torres; Daza, 1996), y

- constitución de una red de centros que potenciara el apoyo profesional entre su personal, la más amplia cooperación bibliotecaria y la solución a los problemas comunes.

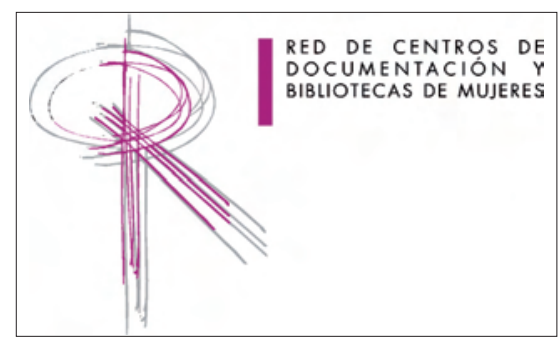

Figura 1. Logotipo de la Red de Centros de Documentación y Bibliotecas de Mujeres

\section{Características y evolución}

La Red no depende de ningún organismo administrativo concreto y tiene su razón de ser en los objetivos de un mejor conocimiento mutuo, apoyo, divulgación, autoformación, coordinación, reivindicación, difusión y mejora del acceso a los recursos informativos y documentales de género, feminismo y mujeres en general. Para poder adherirse a ella los centros interesados deben cumplir unos requerimientos mínimos como: ser de uso público, con un horario determinado de atención al usuario, y disponer de unos fondos documentales específicos, catalogados y organizados con procedimientos normalizados, así como de personal técnico y/o con experiencia en gestión documental.

Después de diecisiete años de reuniones, encuentros, jornadas, etc., nos hallamos ante una Red totalmente consolidada que ha procurado dar forma a una dinámica basa- 
da en la comunicación permanente, ya sea de forma presencial o virtual, junto a la celebración de un encuentro anual donde, además de reforzar el conocimiento y la relación personal y profesional, se intercambian opiniones y propuestas, se presentan las novedades y buenas prácticas de cada centro, se debaten cuestiones de fondo que preocupan, se acuerdan por decisión mayoritaria las principales líneas de actuación a seguir anualmente y se definen los proyectos a llevar a cabo por las comisiones de trabajo de la Red.

\section{"La primera biblioteca de España y Europa dirigida a las mujeres fue la Biblioteca Pública Francesca Bonnemaison (1909)"}

\section{Tipos de centros que integran la Red}

Según su dependencia administrativa los 41 centros $^{2}$ se clasifican en cuatro grupos:

- dependientes de los organismos gubernamentales promotores de políticas públicas para la igualdad, de las administraciones estatal y autonómicas. Representan el 39\% del total;

- bibliotecas y centros de información y recursos de la administración local vinculados a ayuntamientos y diputaciones. Son el $32 \%$;

- bibliotecas de asociaciones afines al movimiento feminista, que a su vez componen, dentro de la Red, la Asociación de Centros de Documentación y Bibliotecas de Mujeres "María Moliner" (Argente-Jiménez, 2009). Esta asociación se creó durante el VEncuentro-Telde, 1998- por parte de las bibliotecas y centros de documentación ligados al movimiento feminista,

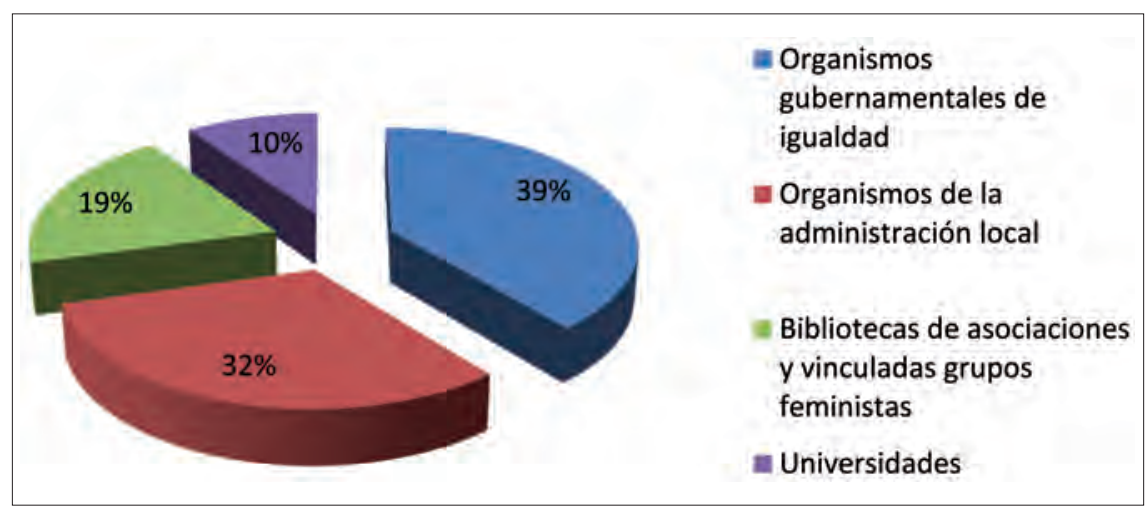

Figura 2. Dependencia de las unidades de información que integran la Red

con la intención de lograr financiación económica para la Red y obtener los recursos necesarios para la realización de actividades mediante la presentación de proyectos sobre género (Fraile, 2000). Contribuyen con el $19 \%$ de los centros.

- servicios de documentación de los centros de investigación universitaria. Con tan sólo 4 centros representan el $10 \%$.

\section{"La Red de Centros de Documentación y Bibliotecas de Mujeres se creó en España en 1995 para difundir la labor de sus integrantes"}

Por lo que respecta a la procedencia geográfica, todas las autonomías españolas están representadas y cuentan con algún centro integrado en la Red. Las comunidades autónomas con más participación son: Catalunya con 8 centros, seguida de Andalucía y Madrid, con 6 cada una; Asturias y la Comunidad Valenciana con 3; Castilla y León, Euskadi y Navarra con 2; y las nueve autonomías restantes con un solo centro cada una de ellas. Hay que destacar la ausencia de centros o servicios de documentación de los organismos gubernamentales promotores de políticas públicas de igualdad de las comunidades autónomas de Aragón, Canarias o Galicia que, aunque en su web publicitan interesantes centros de documentación, hasta el momento no han demostrado interés por incorporarse a la Red, a excepción del Centro de Documentación del Instituto Aragonés de la Mujer que está iniciando su incorporación.

\section{Objetivos generales}

- Fomentar la cooperación profesional entre sus centros;

- coordinar el esfuerzo y la labor que realizan los centros de la Red con vistas a la unidad de acción para un mejor logro de sus fines;

- unificar criterios en el tratamiento de la documentación para facilitar el intercambio de información;

- elaborar herramientas de trabajo, productos documentales, etc., que faciliten la labor de los centros de la Red;

- propiciar la publicidad de la Red y de los centros de documentación y bibliotecas que la integran y de sus fondos;

- favorecer la formación continuada del personal especializado en biblioteconomía y documentación, desde una perspectiva de género, en el uso de las tecnologías y en la gestión de los centros de documentación y bibliotecas de mujeres;

- facilitar el uso y desarrollo de las TIC en el tratamiento y acceso de la información; 


\begin{tabular}{|c|c|c|c|}
\hline \multicolumn{2}{|c|}{ Encuentros } & \multirow{2}{*}{ Organización } & \multirow{2}{*}{ Principales temas tratados } \\
\hline № & $\begin{array}{l}\text { Lugar y } \\
\text { fecha }\end{array}$ & & \\
\hline I & $\begin{array}{l}\text { Pamplona, } \\
30 \text { sep.-2 } \\
\text { oct. } 1994\end{array}$ & $\begin{array}{l}\text { Centro de Documen- } \\
\text { tación y Biblioteca de } \\
\text { Mujeres de IPES (Ins- } \\
\text { tituto de Promoción y } \\
\text { Estudios Sociales) } \\
\end{array}$ & Exposición de problemas comunes y primeros intentos de cooperación. \\
\hline II & \begin{tabular}{|l|} 
Madrid, \\
$6-8$ oct. 1995 \\
\end{tabular} & Biblioteca de Mujeres & Creación de una red y elaboración de un registro de centros. \\
\hline III & $\begin{array}{l}\text { Granada, } \\
4-6 \text { sep. } \\
1996\end{array}$ & $\begin{array}{l}\text { Biblioteca del Instituto } \\
\text { de Estudios de la } \\
\text { Mujer, Universidad de } \\
\text { Granada }\end{array}$ & Definición y perfil de la Red de Centros de Documentación y Bibliotecas de Mujeres. \\
\hline IV & $\begin{array}{l}\text { Barcelona, } \\
17-19 \text { oct. } \\
1997 \\
\end{array}$ & $\begin{array}{l}\text { Centre de Documenta- } \\
\text { ció, Institut Català de } \\
\text { les Dones }\end{array}$ & $\begin{array}{l}\text { Legalización de la Red. } \\
\text { Primeros contactos con las redes internacionales de bibliotecas de mujeres. } \\
\text { Definición del rol de las bibliotecas y centros de documentación de mujeres respecto } \\
\text { a la igualdad de oportunidades entre mujeres y hombres. }\end{array}$ \\
\hline V & \begin{tabular}{|l|} 
Telde-Gran \\
Canaria, \\
1-4 oct. 1998
\end{tabular} & $\begin{array}{l}\text { Ayuntamiento de } \\
\text { Telde (Gran Canaria), } \\
\text { Concejalía de Mujer }\end{array}$ & $\begin{array}{l}\text { Fuentes y métodos para el estudio de la historia de la mujer en Canarias. } \\
\text { Información sobre la Know how conf on the world of women's information } \\
\text { (Amsterdam, agosto 1998). } \\
\text { La mujer en el refranero español. } \\
\text { Creación de la Asociación de Centros de Documentación y Bibliotecas de Mujeres } \\
\text { "María Moliner". }\end{array}$ \\
\hline $\mathrm{VI}$ & \begin{tabular}{|l|} 
Mérida, \\
7-9 oct. 1999
\end{tabular} & $\begin{array}{l}\text { Centro de Estudios y } \\
\text { Documentación de la } \\
\text { Fundación } 8 \text { de Marzo }\end{array}$ & $\begin{array}{l}\text { Experiencias de cooperación y buenas prácticas entre centros oficiales y del } \\
\text { movimiento feminista. } \\
\text { Las librerías de mujeres y la selección bibliográfica en las bibliotecas de mujeres. } \\
\text { Métodos de elaboración del catálogo colectivo de publicaciones periódicas de la Red. }\end{array}$ \\
\hline VII & $\begin{array}{l}\text { Medina del } \\
\text { Campo, } \\
\text { Valladolid, } \\
28-30 \text { sep. } \\
2000\end{array}$ & $\begin{array}{l}\text { Asociación de Mujeres } \\
\text { Rosa Chacel de Valla- } \\
\text { dolid }\end{array}$ & $\begin{array}{l}\text { Propuestas de sistematización de la documentación legislativa de género. } \\
\text { Servicios en internet y listas de distribución sobre mujeres. } \\
\text { Preparación del taller que la Red presentaría en las VII Jornadas Fesabid } 2000 \\
\text { (Bilbao). } \\
\text { Elección del logotipo de la Red. } \\
\text { Directrices de préstamo interbibliotecario. }\end{array}$ \\
\hline VIII & $\begin{array}{l}\text { Vigo, } \\
18-20 \text { oct. } \\
2001\end{array}$ & $\begin{array}{l}\text { Alecrín, Grupo de } \\
\text { Estudios sobre a Con- } \\
\text { dición da Muller }\end{array}$ & $\begin{array}{l}\text { Servicios, propuestas y actividades de centros de la Red. } \\
\text { Fuentes de información sobre mujeres. } \\
\text { Actualización de recursos en internet. } \\
\text { Interpretación de algunos descriptores del Tesauro Mujer. } \\
\text { http://www.inmujer.migualdad.es/mujer/servicios/centro_documentacion/ } \\
\text { Tesauro06. pdf }\end{array}$ \\
\hline IX & $\begin{array}{l}\text { Gijón, 24-26 } \\
\text { oct. } 2002\end{array}$ & $\begin{array}{l}\text { Ayuntamiento de } \\
\text { Gijón, Concejalía de la } \\
\text { Mujer }\end{array}$ & $\begin{array}{l}\text { Archivo de prensa, cd-rom y otros soportes audiovisuales: propuestas de } \\
\text { sistematización y elaboración. } \\
\text { Experiencias de dinamización en bibliotecas de mujeres. } \\
\text { Nuevas perspectivas en centros de documentación: género y desarrollo. } \\
\text { Información sobre la reunión europea de WI NE (Women Information Network } \\
\text { Europe) en Bolonia, septiembre } 2002 \text {. }\end{array}$ \\
\hline $\mathrm{x}$ & $\begin{array}{l}\text { Vitoria, } 23- \\
25 \text { oct. } 2003\end{array}$ & $\begin{array}{l}\text { Centro de Documen- } \\
\text { tación de Emakunde / } \\
\text { Instituto Vasco de la } \\
\text { Mujer }\end{array}$ & $\begin{array}{l}\text { Impacto y rentabilidad de los recursos electrónicos en las bibliotecas y centros de } \\
\text { documentación de mujeres. } \\
\text { Optimización de herramientas para la indización: taller sobre tesauros especializados. } \\
\text { Asiste como invitada una representante de Isis Internacional (Chile), que dio a } \\
\text { conocer aspectos del funcionamiento y organización de este servicio de información y } \\
\text { documentación de prestigio internacional. }\end{array}$ \\
\hline $\mathrm{XI}$ & $\begin{array}{l}\text { Murcia, } \\
1-3 \text { jul. } 2004\end{array}$ & $\begin{array}{l}\text { Centro de Documen- } \\
\text { tación, Instituto de } \\
\text { la Mujer, Región de } \\
\text { Murcia }\end{array}$ & $\begin{array}{l}\text { Terminología de género: sesgos, interrogantes, posibles respuestas. } \\
\text { Informe y taller estadístico sobre el Tesauro Mujer. } \\
\text { Concursos de narrativa: una oportunidad cultural para las mujeres de la Comunidad } \\
\text { Valenciana. }\end{array}$ \\
\hline XII & $\begin{array}{l}\text { Sevilla, } \\
2-4 \text { jun. } 2005\end{array}$ & \begin{tabular}{|l|} 
Centro de Documen- \\
tación "María Zambra- \\
no", Instituto Andaluz \\
de la Mujer \\
\end{tabular} & $\begin{array}{l}\text { Mujeres y ciencia ficción. } \\
\text { Nuevas tecnologías y servicios bibliotecarios a mujeres inmigrantes. } \\
\text { Cómo elaborar el blog de la Red. } \\
\text { Taller sobre indización con perspectiva de género. }\end{array}$ \\
\hline XIII & $\begin{array}{l}\text { Málaga, 19- } \\
20 \text { jun. } 2006\end{array}$ & $\begin{array}{l}\text { Biblioteca del Servicio } \\
\text { Provincial de la Mujer. } \\
\text { Diputación de Málaga }\end{array}$ & $\begin{array}{l}\text { Presentación del trabajo de las comisiones de: publicaciones periódicas, blog/web, } \\
\text { bibliografía básica de género y tesauro. } \\
\text { Invitada: Remedios Zafra: Feminismos e internet, las mujeres ante el reto de la } \\
\text { alfabetización digital. }\end{array}$ \\
\hline XIV & $\begin{array}{l}\text { Alcobendas, } \\
\text { Madrid, } \\
17-19 \text { mayo } \\
2007 \\
\end{array}$ & $\begin{array}{l}\text { Biblioteca Clara Cam- } \\
\text { poamor. Casa de la } \\
\text { Mujer. Ayuntamiento } \\
\text { de Alcobendas }\end{array}$ & $\begin{array}{l}\text { Homenaje a Isabel de Torres, socia fundadora de la Red. } \\
\text { Intercambio de buenas prácticas en la gestión diaria de una biblioteca de mujeres. } \\
\text { Herramientas para mejorar las relaciones interpersonales en la profesión } \\
\text { bibliotecaria. }\end{array}$ \\
\hline $\mathrm{XV}$ & $\begin{array}{l}\text { Palencia, } \\
28-30 \text { mayo } \\
2008\end{array}$ & $\begin{array}{l}\text { Centro de Documenta- } \\
\text { ción para la Mujer en } \\
\text { Castilla y León, Junta } \\
\text { de Castilla y León } \\
\end{array}$ & $\begin{array}{l}\text { Presentación del trabajo de las comisiones. } \\
\text { Elaboración de una bibliografía básica de género. } \\
\text { Propiedad intelectual y derechos de autoría. }\end{array}$ \\
\hline $\mathrm{XVI}$ & $\begin{array}{l}\text { Barcelona, } \\
11-12 \text { jun. } \\
2009\end{array}$ & $\begin{array}{l}\text { Biblioteca Francesca } \\
\text { Bonnemaison }\end{array}$ & $\begin{array}{l}\text { Revisión, actualización de las normas de funcionamiento de la Red y constitución de } \\
\text { la comisión coordinadora. } \\
\text { TIC y documentación feminista. } \\
\text { Actividades de extensión bibliotecaria en las bibliotecas de mujeres. } \\
\text { Cómo gestionar y difundir los recursos documentales en una biblioteca o centro de } \\
\text { documentación sobre mujeres. }\end{array}$ \\
\hline XVII & $\begin{array}{l}\text { Bilbao, } \\
27-29 \text { mayo } \\
2010\end{array}$ & $\begin{array}{l}\text { Centro de Documenta- } \\
\text { ción de Mujeres "Maite } \\
\text { Albiz" / Emakumeen } \\
\text { Dokumentazio Zentrua } \\
\text { "Maite Albiz" }\end{array}$ & $\begin{array}{l}\text { Recuperación de la memoria histórica de las mujeres. } \\
\text { La importancia de los documentos biográficos y la construcción del movimiento } \\
\text { feminista. } \\
\text { Promoción social y visibilización de la Red. } \\
\text { Herramientas de la web } 2.0 \text {. }\end{array}$ \\
\hline
\end{tabular}

Tabla 1. Resumen de los encuentros anuales celebrados por la Red de Centros de Documentación y Bibliotecas de Mujeres ${ }^{7}$ y principales temas tratados 
- promover a escala internacional el intercambio y la comunicación con otras redes similares.

\section{"Los centros de la $R e d$ están especializados en documentación sobre mujeres, son de uso público y cuentan con personal especializado"}

\section{Visión, misión $y$ líneas de actuación}

\section{Visión}

Ser el referente nacional y estatal de apoyo al análisis y la investigación sobre mujeres, feminismos y estudios de género y de la conservación del patrimonio documen- tal, histórico y bibliográfico de las mujeres.

\section{Misión}

Recopilar, sistematizar, conservar y difundir la información sobre las mujeres, las obras de creación escritas por ellas, los documentos referentes a la política estatal y a las autonómicas de igualdad, entre mujeres y hombres y los generados por la investigación, el movimiento feminista y los grupos de mujeres españoles.

\section{Líneas de actuación}

- Promocionar y difundir socialmente los centros de la Red como productores y depositarios

\section{"La Red no depende de ningún organismo administrativo concreto"}

del patrimonio, la memoria y el conocimiento de las mujeres.

- Potenciar vías de colaboración entre los centros de la Red para difundir sus recursos informativos y documentales: préstamo interbibliotecario, productos documentales, etc.

- Potenciar el trabajo en red para mejorar el rol de los centros como agentes sociales de integración de las mujeres en la dinámica política, social y cultural de España.

- Mantener y elaborar proyectos cooperativos de calidad que optimicen la labor de los centros de la Red y faciliten la mayor especialización de su personal.

- Promover el intercambio y la comunicación con otras redes similares a nivel europeo e internacional.

\begin{tabular}{|c|c|c|}
\hline \multicolumn{3}{|r|}{ Análisis DAFO de la Red de Centros de Documentación y Bibliotecas de Mujeres } \\
\hline \multirow[t]{2}{*}{$\begin{array}{l}\text { Análisis } \\
\text { externo }\end{array}$} & Oportunidades & $\begin{array}{l}\text { Aumento de la demanda y el interés por la información y documentación sobre los estudios de las } \\
\text { mujeres, de género y feministas. } \\
\text { Facilidad de acceso a recursos electrónicos y redes telemáticas. } \\
\text { Crecimiento de la documentación y de los trabajos de investigación elaborados con perspectiva de } \\
\text { género. } \\
\text { Situación favorable, a nivel internacional, en cuanto a la sensibilización social e institucional con res- } \\
\text { pecto al colectivo femenino. } \\
\text { Plena incorporación de las mujeres a los campos de la literatura y del ensayo como autoras e investi- } \\
\text { gadoras. } \\
\text { Evolución de la sociedad hacia la participación activa de las mujeres en los entornos político, social y } \\
\text { laboral. }\end{array}$ \\
\hline & Amenazas & $\begin{array}{l}\text { Coyuntura general de restricción presupuestaria y contención del gasto público. } \\
\text { Falta de reconocimiento social de la importancia de las unidades de información sobre mujeres. } \\
\text { Posibles reticencias del movimiento feminista radical hacia la información procedente de los organis- } \\
\text { mos oficiales. } \\
\text { Monopolios editoriales y políticas de propiedad intelectual. } \\
\text { Tradicional resistencia de las mujeres al propio desarrollo personal. } \\
\text { Tecnofobia o rechazo de las mujeres al uso de las TIC y al aprovechamiento de sus potencialidades. }\end{array}$ \\
\hline \multirow[t]{2}{*}{$\begin{array}{l}\text { Análisis } \\
\text { interno }\end{array}$} & Fortalezas & $\begin{array}{l}\text { Uso de las TIC en la gestión, tratamiento de la información y en los servicios a los usuarios. } \\
\text { Especificidad de los fondos, exclusivos en estudios de género, feminismos y mujeres y actualización } \\
\text { constante e inmediata de la documentación producida a escala nacional e internacional. } \\
\text { Edición propia de obras y trabajos de investigación sobre el tema. } \\
\text { Contacto directo con las investigadoras, universitarias, etc., productoras de informes, estudios y traba- } \\
\text { jos de investigación de género, feminismos y mujeres. } \\
\text { Plena motivación y vocación por parte del personal que presta servicios en los centros de la Red. } \\
\text { Red de contactos internacionales al estar integrada en Mapping the World: } \\
\text { http://www.iiav.nl/eng/databases/mapping/index.html } \\
\text { y WINE (Women Information Network Europe): } \\
\text { http://www. women.it/wine/index.htm } \\
\text { Representante de Europa Occidental en el Comité Permanente de la Know how conference on the world } \\
\text { of women's information entre } 1998 \text { y 2002. } \\
\text { http://www.iiav.nl/eng/ic/knowhow/index.html }\end{array}$ \\
\hline & Debilidades & $\begin{array}{l}\text { Recursos humanos insuficientes. } \\
\text { Poca oferta de formación y reciclaje sobre gestión de bibliotecas de mujeres. } \\
\text { Falta de mecanismos de promoción social y marketing. } \\
\text { Restricciones presupuestarias que condicionan la ampliación de recursos y servicios compartidos. } \\
\text { Falta de implicación entre las organizaciones e instituciones de que dependen las bibliotecas y centros } \\
\text { de documentación de mujeres. } \\
\text { Falta de cultura de trabajo en sistemas cooperativos o de colaboración. } \\
\text { Dificultad de definición de un marco legal que aglutine los centros de la Red. }\end{array}$ \\
\hline
\end{tabular}

Tabla 2. Análisis DAFO de la Red de Centros de Documentación y Bibliotecas de Mujeres 


\section{A modo de conclusión}

La Red de Centros de Documentación y Bibliotecas de Mujeres está formada por centros de diversa entidad que dependen de varios proyectos $\mathrm{u}$ organizaciones sociales, académicas, gubernamentales o municipales. Cada uno de ellos se enmarca en entornos de funcionamiento distintos, con características y formas de organización propias. A pesar de esto, la sensibilidad acerca de los temas que se trabajan y el deseo de visibilizar el conocimiento femenino, contribuyendo a erradicar el androcentrismo y la marginación secular a que se han visto sometidas las mujeres, también en el ámbito de la biblioteconomía, son comunes. Ello ha hecho posible la pervivencia de una red de estas características que hubiese sido muy difícil desarrollar en otros ámbitos temáticos. En un mundo globalizado como el actual, donde no puede obviarse la perspectiva del trabajo colectivo, se reafirma una vez más la importancia de la creación de redes de colaboración y coordinación como instrumento para optimizar recursos y aumentar la difusión y dinamización de los centros.

Las bibliotecas y centros de documentación sobre mujeres consti- tuyen uno de los tipos de bibliotecas menos conocidos entre el público en general, y en ese sentido la Red, mediante la participación activa y el trabajo solidario que ha ido llevando a cabo durante los 17 años de vida del proyecto, ha conseguido hacerlas visibles y hacer más fácil el acceso a la información de género, convirtiendo el proyecto, con sus debilidades y fortalezas, en una experiencia rica y satisfactoria que justifica el sentido, aprovechamiento y utilidad de esta Red para todos sus participantes.

\section{"Después de 17 años de Encuentros se tiene una red totalmente consolidada"}

\section{Notas}

1. Cada año se siguen celebrando los Encuentros. El próximo tendrá lugar en 2011 en L'Hospitalet de Llobregat (Barcelona), organizado por el Centre de Documentació del Programa Municipal per a la Dona (Ajuntament de L'Hospitalet).

2. La última actualización del directorio de centros que integran la Red de Centros de Documentación y Bibliotecas de Mujeres puede consultarse en:

http://www20.gencat.cat/docs/icdones/02\% 20Serveis/Arxiu\%20docs/Directorio_Web.pdf

\section{Bibliografía}

Anitua-Vallés, Estibalitz; Argente-Jiménez, Montse; Chinea-Meseguer, Manuel; DazaBonachela, Aure. "Bibliotecas de mujeres: unas grandes desconocidas". Mi biblioteca: la revista del mundo bibliotecario, primavera 2007, n. 9, pp. 106-115.

Argente-Jiménez, Montse. "Bibliotecas de mujeres". Anuario de bibliotecas españolas 2008. Málaga: Fundación Alonso Quijano, 2008, pp. 302-305.

Argente-Jiménez, Montse. "Bibliotecas de mujeres". Anuario de bibliotecas españolas 2009. Málaga: Fundación Alonso Quijano, 2009, pp. 274-277.

De-Torres, Isabel; Daza, Aure. Guía de bibliotecas y centros de documentación de mujeres en España 1996. Sevilla: Instituto Andaluz de la Mujer, 1996. ISBN 84-7921-044-3.

Fraile, María-Ángeles. "La Asociación 'María Moliner"'. Métodos de información, 2000, enero-marzo, v. 7, n. 35-36, pp. 81-84.

http://www.avei.org/revista/mei35/2000-3581.pdf

Ana M. Muñoz-Muñoz. Departamento de Biblioteconomía y Documentación, Facultad de Comunicación y Documentación. Universidad de Granada.

anamaria@ugr.es

Montse Argente-Jiménez. Centre de Documentació, Institut Català de les Dones, Departament d'Acció Social i Ciutadania, Generalitat de Catalunya.

margente@gencat.cat

\title{
Próximos temas centrales
}

\author{
Noviembre 2010 \\ Medios de comunicación en internet \\ Enero 2011 \\ Psicología y sociología de la información \\ Marzo 2011 \\ Archivos administrativos e intranets \\ Mayo 2011 \\ Información de las administraciones públicas \\ Julio 2011 \\ Fotografía y bancos de imágenes \\ Septiembre 2011 \\ Inteligencia competitiva
}

Los interesados pueden remitir notas, artículos, propuestas, publicidad, comentarios, etc., sobre estos temas a: http://recyt.fecyt.es/index.php/EPI/index 